\title{
SAMPLE EXTRACTION BSAED ON HELIX SCATTERING FOR POLARIMETRIC SAR CALIBRATION
}

\author{
Yonglei Chang a, Jie Yang a, *, Pingxiang Li $^{\text {a }}$, Lingli Zhao ${ }^{\text {b, c }}$, Lei Shi ${ }^{\text {a }}$, \\ ${ }^{a}$ State Key Laboratory of Information Engineering in Surveying, Mapping and Remote Sensing, University of Wuhan, Luo Yu Road \\ No.129, Wuhan, China, 430079 \\ ${ }^{\mathrm{b}}$ School of Remote Sensing and Engineering, University of Wuhan, Luo Yu Road No.129, Wuhan, China, 430079 \\ ${ }^{\mathrm{c}}$ Beijing Key Laboratory of Urban Spatial Information Engineering \\ Email: changyonglei@whu.edu.cn Tel: +86-15623037628
}

Commission III, WG III/6

KEY WORDS: SAR, Helix scattering, Polarimetric Calibration, Reflection symmetry, Thresholding Segmentation

\begin{abstract}
:
Polarimetric calibration (PolCAL) of Synthetic Aperture Radar (SAR) images is a significant preprocessing for further applications. Since the reflection symmetry property of distributed objects can provide stable constraints for PolCAL. It is reasonable to extract these reference samples before calibration. The helix scattering generally appears in complex urban area and disappears for a natural scatterer, making it a good measure to extract distributed objects. In this paper, a novel technique that extracts reflecting symmetry samples is proposed by using helix scattering. The helix scattering information is calculated by Yamaguchi four-component decomposition algorithm. An adaptive threshold selection algorithm based on generalized Gaussian distribution is also utilized to scale the helix scattering components automatically, getting rid of the problem of various numerical range. The extracting results will be taken as PolCAL reference samples and the Quegan method are utilized to calibrate these PolSAR images. A C-band airborne PolSAR data was taken as examples to evaluate its ability in improving calibration precision. Traditional method i.e. extracting samples with span power was also evaluated as contrast experiment. The results showed that the samples extracting method based on helix scattering can improve the Polcal precision preferably.
\end{abstract}

\section{INTRODUCTION}

A polarimetric synthetic aperture radar (PolSAR) system measures the scattering power from terrain with different polarimetric waves, recording abundant information of ground objects. However, before the recorded images being used for further research or application, polarimetric calibration (PolCAL) is needed to ensure the relative amplitude and phase between different image channels (van Zyl, 2011). A lot of research on PolCAL have been done since the first PolSAR system i.e. AIRSAR was developed. At first, different kinds of Corner Reflectors (CR) and Polarimetric Active Radar Calibrator (PARC) were utilized and most of the PolCAL research were based on them (Whitt, 1991). Then, some particular scattering targets like bare soil, desert, grassland, were investigated and taken as PolCAL references (Sarabandi, 1994). The presupposition of SAR system was also exploited (van Zyl, 1989). Currently, most of the PolCAL algorithms take distributed targets as calibration samples to calculate Crosstalk parameters and cross polarized channel imbalance (Cross-Imba) parameter, and use at least one $\mathrm{CR}$ to calculate the copolarized channel imbalance (Co-Imba) parameter. PolCAL method without CR was also proposed, and it can be taken as an emergency treatment when CR is not available (Shi, 2014).

The distributed targets provide stable polarization constrains for PolCAL, i.e. reflecting symmetry and reciprocal scattering. The

* Corresponding author

Jie Yang, PhD., professor. Email: yangj@whu.edu.cn
Quegan PolCAL methods use both of these two constrains formulating PolCAL equations (Quegan, 1994; Kimura, 2004). To get better calibration accuracy, it is reasonable to extract distributed target samples before calibration. For the present PolSAR systems, the reciprocal scattering is a general knowledge because of the monostatic backscattering case. The reflecting symmetry target usually appears in distributed area, like forest, desert, bare soil, or farmland. The widely used methods of extracting distributed samples are performed by scaling the span or polarimetric coefficients of the entire image, which is very sketchy and artificial. The helix scatterer generally appears in complex urban area and disappears for natural distributed scatterer (Yamaguchi, 2005). Based on this property, we proposed a novel method of PolCAL samples extraction. An automatic thresholding procedure is also applied to solve the various value field problem, which arises from the uncalibrated data of different systems.

\section{POLARIMETRIC CALIBRATION METHOD}

\subsection{The Polarimetric Distortion Model}

During the PolSAR observation, the real scattering coefficients of terrain will be affected by polarimetric distortions, and the distortions are Crosstalk, Cross-Imba, Co-Imba, System Noise and Faraday rotation. The Faraday rotation will be neglected for airborne systems. Furthermore, with System Noise simplified, 
the relationship between the measured data and the real scattering coefficients will be formulated as:

$$
\begin{aligned}
& {\left[\begin{array}{l}
O_{h h} \\
O_{h v} \\
O_{v h} \\
O_{v v}
\end{array}\right]=Y\left(\begin{array}{cccc}
1 & v & w & w v \\
z & 1 & w z & w \\
u & u v & 1 & v \\
u z & u & z & 1
\end{array}\right)\left(\begin{array}{cccc}
\alpha & 0 & 0 & 0 \\
0 & \alpha^{-1} & 0 & 0 \\
0 & 0 & \alpha & 0 \\
0 & 0 & 0 & \alpha^{-1}
\end{array}\right)} \\
& \left(\begin{array}{llll}
k & 0 & 0 & 0 \\
0 & 1 & 0 & 0 \\
0 & 0 & 1 & 0 \\
0 & 0 & 0 & k^{-1}
\end{array}\right)\left[\begin{array}{l}
S_{h h} \\
S_{v h} \\
S_{h v} \\
S_{v v}
\end{array}\right]+N\left[\begin{array}{l}
1 \\
1 \\
1 \\
1
\end{array}\right] \\
& \Rightarrow[O]=Y[X][Q][K][S]+[N]
\end{aligned}
$$

(1)

The matrix $\vec{O}$ is the observed scattering vector, $\vec{S}$ stands for the real scattering vector of targets. The matrixes $X, Q, K$ are the Crosstalk, Cross-Imba, Co-Imba separately. Scalar $N$ is the system noise, and $Y$ is the absolute calibration parameter.

\subsection{The Quegan PolCAL Algorithm}

The Quegan PolCAL algorithm is efficient and stable. Unlike the Ainsworth algorithm, Quegan's calculation results seldom have distortion problem, so they can express the polarimetric condition of SAR systems more veritably (Ainsworth, 2006; Xing, 2012). Besides the Quegan method, the reflecting symmetry assumption is widely used in PolCAL. Under this assumption, the complex correlation coefficients between co-polarization and cross polarization are negligible, and the covariance matrix of these scatterer is shown below:

$$
[C]_{\text {real }}=\left(\begin{array}{ccc}
\left\langle S_{h h} S_{h h}^{*}\right\rangle & 0 & \left\langle S_{h h} S_{v v}^{*}\right\rangle \\
0 & \left\langle S_{h v} S_{h v}^{*}\right\rangle & 0 \\
\left\langle S_{v v} S_{h h}^{*}\right\rangle & 0 & \left\langle S_{v v} S_{v v}^{*}\right\rangle
\end{array}\right)
$$

The polarimetric distortion parameters will make the correlation coefficients in the observed covariance matrix more visibly. Finally, the distortion parameters will be calculated out by adding some other assumptions.

\section{POLCAL SAMPLES EXTRACTION}

\subsection{Reflecting Symmetry Scattering Samples}

Since the PolCAL algorithms are based on reflecting symmetry scattering, the extraction of calibration samples is necessary before parameter calculation. In the Yamaguchi four-component decomposition algorithm, helix component is added in to account for the non-reflecting symmetry scattering case. While for the distributed arear that satisfied the reflecting symmetry condition, the helix scattering will disappear. In this paper, the helix scattering is investigated and scaled. Scatterer with higher helix component will be masked off and the lower ones will be regarded as symmetric scattering samples.

In coherent decomposition, the helix scattering is calculated by the circular polarization basis transformation. The differences between $S_{L L}$ and $S_{R R}$ will be regarded as helix component. While in the PolCAL, the covariance matrix will be taken as the input data and the calibration samples need to be incoherent averaged. Therefore, the calculation of helix scattering component should be performed by incoherence methods. After the speckle noise suppression of the uncalibrated PolSAR data, the helix scattering component is calculated as below:

$$
P_{\text {helix }}=\left|\operatorname{Im}\left(\left\langle S_{H V}^{*}\left(S_{H H}-S_{V V}\right)\right\rangle\right)\right|
$$

\subsection{Automatic Thresholding Segmentation}

For most PolSAR calibration scheme, the radiometric calibration comes after the PolCAL. As a result, the images needed to be PolCAL are usually have not been processed by absolute radiometric calibration. Furthermore, PolSAR system designers are more likely to use unique data storage format and value field. Thus, automatic threshold segmentation should be performed after helix component calculation. The Kittler-Illingworth (KI) threshold selection criterion is widely used in change detection with SAR images. Based on the minimum error decision rule, the $\mathrm{KI}$ criterion function is showed below:

$$
J(T)=\sum_{H_{l}=0}^{L-1} h\left(H_{l}\right) C\left(H_{l}, T\right)
$$

The $H$ stands for the helix component value in image, and $h(\cdot)$ is the static histogram. The $C(\cdot)$ stands for the cost function:

$$
C\left(X_{l}, T\right) \begin{cases}2 \ln \left[\frac{P\left(H_{l}\right)}{P\left(g_{\text {class }}\right) P\left(H_{l} \mid g_{\text {class }}, T\right)}\right] \quad \text { if } & X_{l} \leq T \\ 2 \ln \left[\frac{P\left(H_{l}\right)}{P\left(g_{\text {class } 2}\right) P\left(H_{l} \mid g_{\text {class } 2}, T\right)}\right] \quad \text { if } & X_{l}>T\end{cases}
$$

To fit the distribution of helix component value more properly, the General Gaussian Distribution is deployed. In the equation(5), $T$ is the threshold that needs to be evaluated, and $P(\cdot)$ is the probability. The $g_{\text {class } 1}, g_{\text {class } 2}$ are the two classes after segmentation by threshold $T$.

\section{EXPERIMENT}

\subsection{Test Data and Extraction Results}

To verify the efficiency of the proposed method, we made an experiment with an airborne image. The traditional method was also tested for comparison and analysis. The multiband polarimetric SAR system that developed by IECAS (Institute of Electronics, Chinese Academy of Sciences) acquired X- and Cband data sets over the Zunhua test site in August 2015. Several dihedral and trihedral corner reflectors were deployed for calibration during each observation. Figure 1 shows the PauliRGB image of the test data. Most of the area was farmland. Some of the farmland was covered with crops and part of it was bare soil. There were also groves, buildings, pond in the image. 


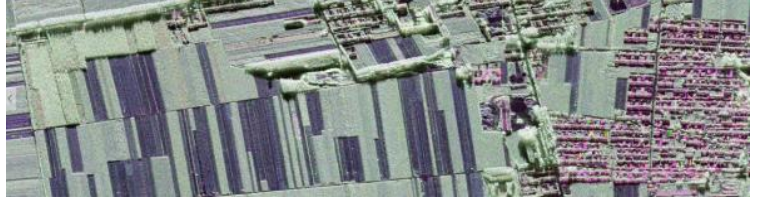

Figure 1. Airborne C-band Pauli-RGB image over Zunhua in August 2015. The top row is the nearest range.

The automatic extracting result of reflecting symmetry sample is showed in Figure 2. In the binary image, the regions with white color were pixels that used for calibration calculation. Generally, farmlands covered with bare soil or crop are typical reflecting symmetry scatterer, while the residential area and grove land scatter unsymmetrically. The extracting result is in good agreement with this experience. Figure 3 shows the extraction result with Span method.

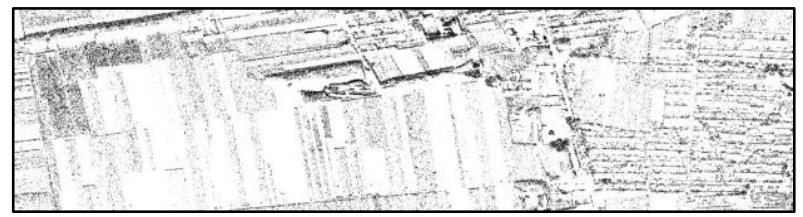

Figure 2. Automatic extracting result of reflecting symmetry sample.

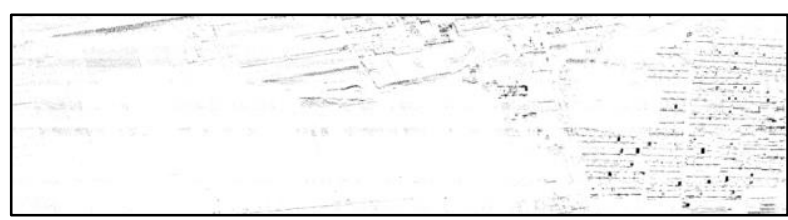

Figure 3. Automatic extracting result with Span method.

\subsection{PolCAL Evaluation and Analysis}

The extracting results were taken as calibration samples for Quegan PolCAL algorithm. Figure 4 shows the polarization responses of trihedral corner reflector. Before PolCAL, the polarization response is quit distorted from the theoretical one. After Quegan PolCAL, both these two method could get good results, and the polarization responses are both closed to the theoretical one. The efficient of these two method need to be analyzed quantitatively.

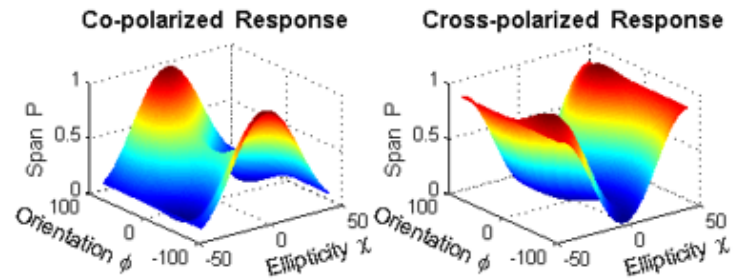

(a) Before PolCAL

Co-polarized Response
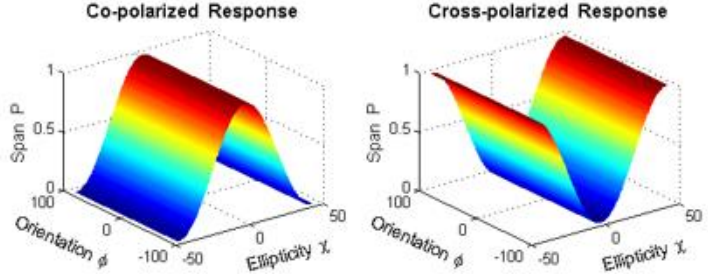

(b) The theoretical

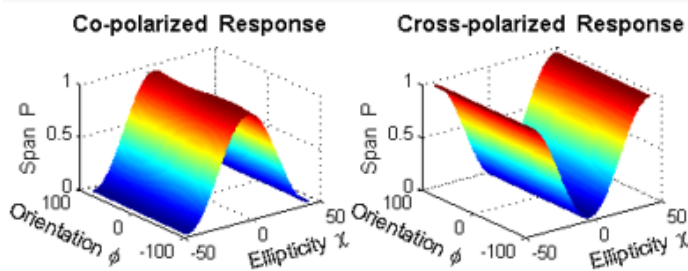

(c) Proposed method

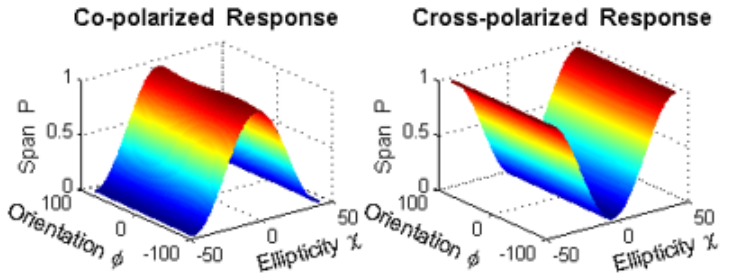

(d) Span method

Figure 4. Polarization response of trihedral corner reflector.

After PolCAL, the calibrated images still have residual polarimetric error, and the magnitude of residual error stands for PolCAL accuracy. In the experiment, we used Ainsworth method to calculate the residual error in the calibrated image. The residual error is showed in Figure 5. In the ideal case, the Alpha equals to 1 , and the Crosstalk is zero. We can see that the residual error of the proposed method is smaller.

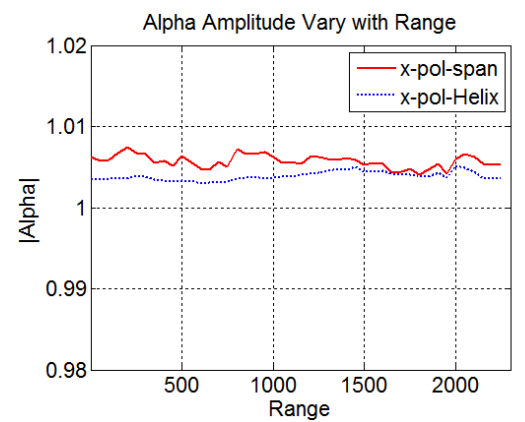

(a) The residual error of Alpha

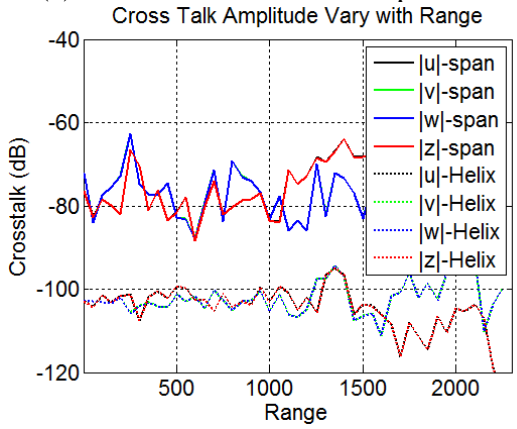

(b) The residual error of Crosstalk

Figure 5. The residual error in image after Quegan PolCAL

\section{CONCLUSION}

A new calibration samples extracting method is proposed based on helix scattering and automatic threshold segmentation. The experiment shows that the new method can improve the accuracy of SAR polarimetric calibration. This method can provide calibration samples for PolCAL algorithms that based on reflecting symmetry scattering. 


\section{ACKNOWLEDGEMENT}

The authors would like to thank the Institute of Electronics, Chinese Academy of Sciences (IECAS) for providing the data in this study. The research is founded by the Land Surface Recognition Technology with GF-3 Satellite Image, No. 03Y20A10-9001-15/16; the National Natural Science Foundation of China, No. 41501382; No. 61371199, Beijing Key Laboratory of Urban Spatial Information Engineering, NO. 2017215.

\section{REFERENCES}

Ainsworth T.L., Ferro-Famil L., Lee J.-S., 2006. "Orientation angle preserving a posteriori polarimetric SAR calibration," Geoscience and Remote Sensing, IEEE Transactions on, vol.44, pp.994-1003.

Kimura H., Mizuno T., Papathanassiou K.P., Hajnsek I., 2004. Improvement of polarimetric SAR calibration based on the Quegan algorithm, IEEE, International Geoscience and Remote Sensing Symposium Proceedings.

Quegan S., 1994. "A unified algorithm for phase and cross-talk calibration of polarimetric data-theory and observations," Geoscience and Remote Sensing, IEEE Transactions on, vol.32, pp.89-99.

Sarabandi K., 1994. "Calibration of a polarimetric synthetic aperture radar using a known distributed target," IEEE Transactions on Geoscience \& Remote Sensing, vol.32, pp.575 582.

Shi L., Yang J., Li P., 2014. "Co-polarization channel imbalance determination by the use of bare soil," ISPRS Journal of Photogrammetry and Remote Sensing, vol.95, pp.53-67.

Van Zyl J.J., 1989. "A technique to calibrate polarimetric radar images using only image parameters and trihedral corner reflectors," Quantitative Remote Sensing: An Economic Tool for the Nineties, Volume 1, vol.1, pp.2889-2892.

Van Zyl J.J., 2011. Synthetic aperture radar polarimetry. John Wiley \& Sons.

Whitt M.W., Ulaby F.T., Polatin P., Liepa V.V., 1991. "A general polarimetric radar calibration technique," Antennas and Propagation, IEEE Transactions on, vol.39, pp.62-67.

Xing S.-Q., Dai D.-H., Liu J., Wang X.-S., 2012. "Comment on "Orientation Angle Preserving A Posteriori Polarimetric SAR Calibration"," IEEE Transactions on Geoscience and Remote Sensing, vol.50, pp.2417-2419.

Yamaguchi Y., Moriyama T., Ishido M., Yamada H., 2005. "Fourcomponent scattering model for polarimetric SAR image decomposition," Geoscience and Remote Sensing, IEEE Transactions on, vol.43, pp.1699-1706. 